\title{
Standard of Molecular Dynamics Modeling and Simulation of Relaxation in Dense Media
}

\author{
Alexey Y. Kuksin, Igor V. Morozov, Genri E. Norman, and \\ Vladimir V. Stegailov \\ Institute for High Energy Densities, Russian Academy of Sciences \\ IHED-IVTAN, Izhorskaya 13/19, Moscow 125412, Russia \\ bogous@orc.ru
}

\begin{abstract}
Approaches to simulation and modeling of relaxation in dense media are developed which would be universal for some classes of relaxation processes. Three examples of relaxation in strongly dissimilar multi-scale systems are considered: equilibration of electrons and ions in a nonisothermal nonideal plasma, lifetime and nucleation of crystals at superheating, void formation in a crystal under negative pressures.
\end{abstract}

\section{Introduction}

Molecular dynamics (MD) method could be a powerful tool for studying nonequilibrium states and relaxation phenomena in dense many-particle systems. In this connection we would mention studies of melting front velocity [1], damping oscillation regime in one-component nonideal plasmas [2], relaxation to equilibrium in two-component two-temperature nonideal plasmas [3] , recombination relaxation in ultracold plasmas [5], spontaneous decay of a superheated crystal [6], relaxation of $S H$ radical in solid krypton [7, equilibration and sampling of a biomolecule [8], protein folding [9], relaxation in shock wave front [10].

Modeling and simulation of relaxation is a relatively new sector of computer science where the standard of approaches, models and numerics has not been established. This paper contribute to the development of this standard. In section 2 approaches are considered which are specific for the problem studied. They are different from those used in modeling and simulation of equilibrium dense media. Some examples of our results are presented in section 3 which validate the approaches developed. The examples chosen correspond to multi-scale systems: two timescales in plasma due to the electron-ion mass ratio, short and long relaxation stages in plasma due to dynamics and stochastic regimes; the lifetime and the decay duration in metastable crystals which are different by several orders of magnitude.

\section{Standard}

Any conventional MD simulation starts from more or less arbitrary initial conditions. Then different approaches are applied to equilibrate the system. Only 
subsequent equilibrium run is used to obtain the useful information. The objective of the simulation of relaxation is of the opposite sense, i.e. to get the information from the non-equilibrium part of the MD run, from that part which is discarded and is not used in equilibrium MD simulations. It is evident, that the requirements to the simulation and modeling of the relaxation should differ from those for the equilibrium ones.

Initial state. The choice of the initial state cannot be an arbitrary one. The initial state should correspond to the physical problem we would like to study. The physical system is modeled with respect to the boundary conditions, the character of non-equilibrium and the minimum particle number which is required to reproduce in simulation the main features of the relaxation process studied.

Ensemble of initial states. One initial state is not sufficient as a rule to achieve the satisfactory accuracy. So an ensemble of initial states is needed to obtain a number of MD runs and perform the averaging of the results. Each state in the ensemble should differ from each other significantly but all of them are to be equivalent with respect to the non-equilibrium problem studied. The last requirement needs some art and can be checked by the following criteria. The increase of the number $I$ of initial states increases the accuracy of averaging as $\sqrt{I}$ and does not shift the mean value.

Ensemble-dependence. Non-equilibrium states can be different for the same media. Different ensembles of initial state correspond to those cases in the simulation. The problem is if there are some features of the relaxation processes which are ensemble-independent.

Dynamic and stochastic relaxation. The inherent feature of MD dynamical system is the Lyapunov instability. Due to this instability there appears a dynamic memory time $t_{m}$ which limits the time interval when the Caushy problem is valid for MD numerical integration. For times greater than $t_{m} \mathrm{MD}$ trajectory "forgets" its initial conditions and ceases to correlate with the hypothetical Newtonian trajectory with the same initial conditions [112]. We expect that the duration of ensemble-dependent part of the relaxation correlates with $t_{m}$.

Number of particles. Correlation lengths do not remain constant during the relaxation. The account of these lengths is of a great importance when the cooperative phenomena are considered, e.g. nucleation or plasma waves. The MD box size should be greater than the maximum correlation length which might appear during the relaxation process.

Instantaneous diagnostics. New procedures are to be used to find characteristics of relaxation at a given moment of time, e.g. parameters which qualitatively show the overall degree of deviation from the Maxwellian velocity distribution and from the Gaussian distribution of crystal particles in space. The distribution form shows the details of the deviations. The diagnostics can be applied separately to different regions in space for non-homogeneous cases.

Time-averaged diagnostics. Averaging over a relatively long period is also needed to obtain e.g. the values of dynamic structure factor. The procedures are suggested [4,13] which imply freezing of the instantaneous non-equilibrium state for the period of measurement. The idea is to introduce the energy exchange 
with an external bath keeping constant the non-equilibrium excitation. It is necessary to check that this procedure does not transform the non-equilibrium state during the period of measurement. Then the external source is switched off and the relaxation continues as if there were no period of freezing.

Numerics. Numerical integration with a variable time step is applied if the relaxation includes dramatic increase of velocities of some particles [5]. Pair distribution function is a sensitive tool to detect particles which could approach potentially forbidden small distances due to insufficiently small time step.

Parallel computations. As well known, MD code is hard to parallelise because of many network communications required for the force calculation procedure. An alternative parallelising algorithm is possible in case of simulation of the relaxation. Since the averaging over initial states is required the relaxation from different initial states can be calculated in parallel. The network load is very low in this case. Therefore this algorithm should be used to calculate the relaxation whereas the standard one to obtain an ensemble of initial states (e.g. in subsection 3.1.

Analytics. Relaxation process can be too long to be simulated by MD in particular for multi-scale systems. Models are used in this case. The results obtained are rescaled with the help of analytic expressions which should be found.

\section{$3 \quad$ Validation of Standard}

\subsection{Relaxation in Two-Component Nonideal Plasmas}

We consider a fully-ionized two-component plasma of $N$ electrons and $N$ ions with masses $m$ and $M$, respectively. The ions are singly ionized. The formation of the bound states should be prohibited in classical MD simulations of the systems of free charges by the appropriate choice of the interaction pseudopotential. We use the so called "corrected Kelbg" pseudopotential 14. As low bound states are excluded the pseudopotential does not depend on the chemical element. The number of ions in present simulations is $N=64-800$. The choice of $N$ and another details of simulations are discussed elsewhere [4]11.

The initial nonequilibrium state is characterized by different temperatures of electrons $T_{e}$ and ions $T_{i}$. We used the initial conditions where the velocities of electrons or/and ions are equal to zero. There are also two variants of the initial spacial configuration of the cold ions. The first variant is a crystal structure with cubic lattice which corresponds to the state after ionization in solids. The second one is a quasi-random configuration obtained from the equilibrium isothermal electron-ion plasma with the same number density.

The results of MD runs are averaged over an ensemble of $I=50-200$ initial states. Provided the result is $N$-independent, the relative error is given by $1 / \sqrt{N I}$. The error bars in figures below correspond to the confidence coefficient 0.68. The bars are not indicated if they are smaller than the size of the points. The values of $T_{e}$ and $T_{i}$ are obtained as the average kinetic energy of the particles 

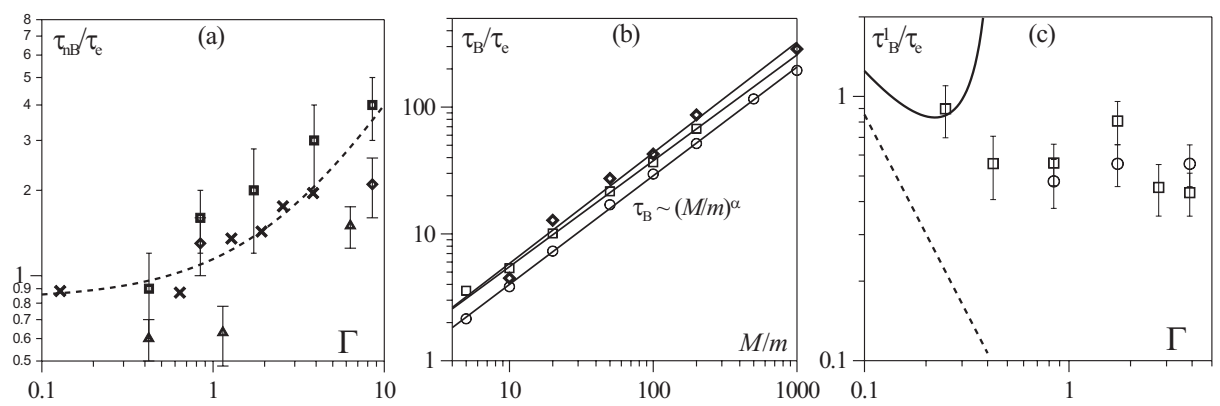

Fig. 1. The dependencies of the duration of the nonexponential relaxation stage $\tau_{n B}$ on the nonideality parameter (a) and of the exponential relaxation time on the mass ratio (b) and on the nonideality parameter (c) for different initial conditions: $T_{i}(0)=$ 0 (crystal) - circles, $T_{i}(0)=0$ (quasirandom) - squares, $T_{e}(0)=0$ - rhombus, $T_{e}(0)=T_{i}(0)=0$ - triangles.

(a) The dynamical memory time $t_{m}$ - crosses, the dashed line is drawn through the crosses to guide the eye.

(a) Straight lines correspond to the power fits $\tau_{B} \sim(M / m)^{\alpha} . \Gamma=1.28$.

(c) Solid curve corresponds to the Landau formula (1), dashed curve - the same with the fixed Coulomb logarithm $L_{e}=3.2$

$T(t)=\frac{1}{2 N I} \sum_{j, k}^{N, I} m v_{j k}^{2}(t)$. The relaxation is charactarized by the the decrease of the difference of electron and ion temperatures $\Delta T=\left|T_{e}-T_{i}\right|$ which following the Boltzmann equation has the exponential asymptote $\Delta T \sim \exp \left\{-t / \tau_{B}\right\}$.

Nonexponential relaxation. The distinguishing feature of a nonideal plasma is a nonexponential stage of the relaxation which precedes the exponential one. The duration of this stage $\tau_{n B}$ does not depend on the mass ratio for $M / m \geq 100$. It increases with the increase of the nonideality parameter (Fig. $1 \mathrm{1}$ ),$\tau_{e}=2 \pi / \omega_{p}$, $\omega_{p}=\sqrt{4 \pi n_{e} e^{2} / m}$ is the period of electron plasma oscillations, $n_{e}$ is the electron number density. The plasma nonideality is characterized by the parameter $\Gamma=$ $e^{2}\left(4 \pi n_{e} / 3\right)^{1 / 3} /\left(k_{B} T\right)$, where $T$ is the final temperature at the end of relaxation. As seen $\tau_{n B}$ depends on the initial conditions but stays comparable with $t_{m}$.

The kinetic equation used to calculate relaxation in ideal plasma implies that the collisions are statistically independent and the particle motion is stochastic. However the time of the stochastization or dynamical memory time $t_{m}$ in nonideal plasma becomes greater than the time between collisions [11.

Dependence of the exponential relaxation time $\tau_{B}$ on plasma properties. The dependence of $\tau_{B}$ on the mass ratio is shown in Fig. 1 b. As seen, different initial conditions result in close values of $\tau_{B}(M)$. The lower relaxation time for the crystal-like initial configuration of ions is caused by an additional heating of ions due to reconfiguration and correlation build up in the ionic subsystem. The mass dependence can be fitted by the power fit $\tau_{B} / \tau_{e} \sim(M / m)^{\alpha}$ in all cases in Fig. 1b. The dependence of $\alpha$ on the nonideality parameter can be fitted by the parabolic curve $\alpha(\Gamma)=1-0.15 \Gamma+0.035 \Gamma^{2}, 0<\Gamma<4$. 
The dependencies of $\tau_{B}$ on the ion mass and $\Gamma$ can be separated as $\tau_{B}(\Gamma, M)=\tau_{B}^{1}(\Gamma)(M / m)^{\alpha(\Gamma)}$. The dependence of $\tau_{B}^{1}(\Gamma)$ is presented in Fig. 11. As seen the initial conditions do not affect $\tau_{B}^{1}$ significantly. In the weak nonideality region the MD results are in a good agreement with the Landau theory [15]

$$
\tau_{B}^{*}=\frac{3}{16} \frac{\left(m T_{i}+M T_{e}\right)}{\sqrt{2 \pi m M}} \frac{1}{e^{4} n_{e} L_{e}},
$$

where $L_{e}$ is the Landau logarithm. The deviation becomes crucial with the increase of $\Gamma$.

One can estimate the relaxation times in real experimental conditions using given $\Gamma$-dependence and mass-dependence. The error of determination of coefficients $\alpha_{B}$ and $\alpha_{n B}$ is about $\xi_{\alpha}=5 \%$. The corresponding error of the extrapolation of relaxation times e.g. for aluminium is $\xi_{\tau}=\log \left(M_{r} / M\right) \xi_{\alpha}=40 \%$, where $M_{r} / M$ is the ratio between real and model ion masses. The obtained precision is enough for comparison by an order of magnitude. The results of such extrapolation correlate with the experimental data [16].

\subsection{Decay of Metastable Crystals}

A system under study is a $f c c$ crystal of particles interacting via Lennard-Jones potential $U(r)=4 \epsilon\left((\sigma / r)^{-12}-(\sigma / r)^{-6}\right)$. Periodic boundary conditions are used.

Decay of crystal subjected to constant rate heating. Superheated solid is a state of matter that can be realized only under particular conditions of high rate energy impacts and/or very low concentration of defects and impurities which could lead to heterogeneous melting (e.g. see [17/18 19]).

Particle structure is prepared for the simulation to be an ideal lattice initially. The system is equilibrated for $10^{4}$ time steps at the temperature $T=0.04 \epsilon$ (extremely low temperature is chosen to preserve the crystal structure completely defect-free). The particular value of temperature is not crucial for the following. The ensemble of initial states can be chosen from the equilibrium run.

Then the model crystal is subject to the isochoric heating at the constant rate $\dot{T}=6 \times 10^{-4}\left(\epsilon^{3} / m \sigma^{2}\right)^{1 / 2}$. The heating is performed by velocity rescaling in the spirit of the Berendsen thermostat technique. As heating is being done the temperature of the model crystal becomes higher than the melting temperature at the given density $T_{m}=1.25 \epsilon$. When temperature reaches $T=1.52 \epsilon$ crystal structure decays into liquid. Phase transformation leads to the step in the dependence of the averaged potential energy $U$ on time $t$ (see Fig. 2).

Structural changes are well manifested in terms of the Lindemann parameter $\delta_{L}=\left\langle\Delta r^{2}\right\rangle / r_{n n}$, where $\left\langle\Delta r^{2}\right\rangle$ is the displacement of particles from the ideal $f c c$ lattice sites averaged over all the particles in the simulation box at the current time step, $r_{n n}$ is the nearest neighbour distance in the lattice. According to the Lindemann criterion of melting $\delta_{L}=0.12-0.13$ for simple crystals at the melting temperature $T_{m}$. This relation holds for the case considered (Fig. 2). However at $T=T_{m}$ there is no phase transition but the crystal becomes superheated and $\delta_{L}$ achieves the values of 0.4 before decay. 

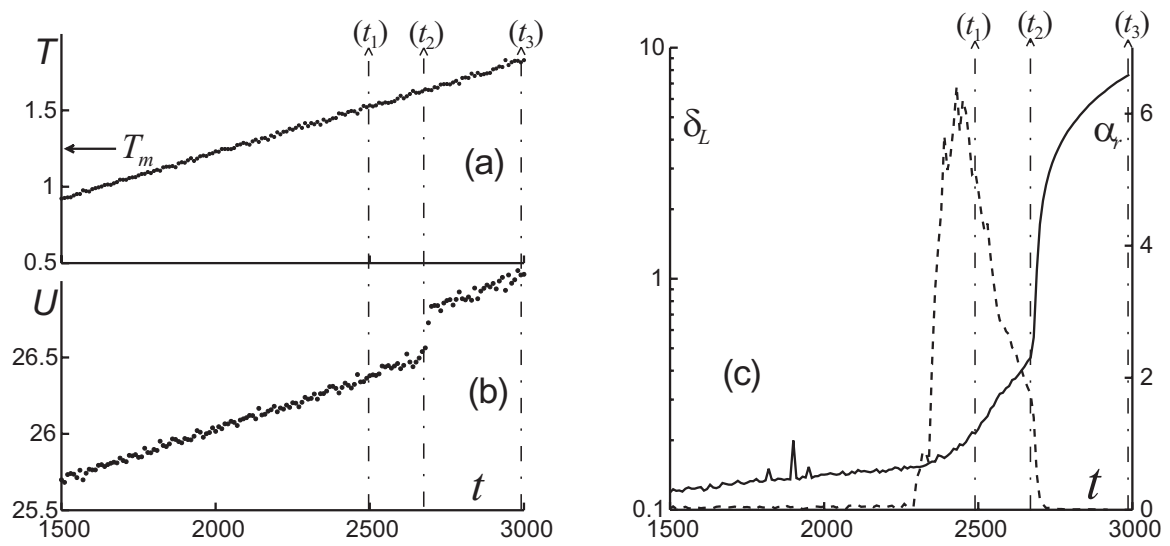

Fig. 2. The time-dependencies: (a) the temperature $T$, (b) the average potential energy of particles $U,(\mathrm{c})$ the Lindemann parameter $\delta_{L}$ (solid) and the non-Gaussian parameter $\alpha_{r}$ (dashed). The time is given in units $\left(m \sigma^{2} / \epsilon\right)^{1 / 2} \cdot N=6912 ; \rho \sigma^{3}=1$

Another parameter $\alpha_{r}=0.6\left(\left\langle\Delta r^{4}\right\rangle /\left\langle\Delta r^{2}\right\rangle^{2}\right)-1$ shows the degree of deviation of the distribution of displacements from the Gaussian shape $\left(\alpha_{r}=0\right.$ for the Gaussian distribution). There is a peak on the $\alpha_{r}$ dependence on time which corresponds to the emergence of collective modes in the motion of particles. This phenomenon can be considered as a precursor of decay under heating. It does not produce any effect on the potential energy time dependence, however Lindemann parameter turns to the faster increase during this peak. After the decay motion of particles becomes irregular, so that $\alpha_{r}$ vanishes and $\delta_{L}$ gets the diffusion-like dependence on time. The loss of long range order is well seen in the evolution of the radial distribution function (in an animation).

The system evolution can be divided into three parts: 1) heating and superheating up to the appearance of the melting precursor; 2) the emergence of collective modes in the particle motion; 3) the decay to the liquid state. The process of the relaxation in the case of the superheated crystal decay is connected with homogeneous nucleation that allows to apply the formalism of the classical nucleation theory (see 206 for details).

Void formation in crystals under negative pressure. A condensed matter can exist at negative pressures. Such states were observed experimentally, e.g. in [21].

Negative pressure states are the metastable ones, so they can exist only a limited period of time. Then the states decay spontaneously and voids are formed. The temperature $T$ and number density $\rho$ are chosen for the simulation to obtain a state near spinodal in order its lifetime can be reached during the MD run. Initial velocities are taken from Maxwellian distribution.

The results are presented for certain examples in Fig. 3.4. Time dependencies for $T$, pressure $P$, parameters $\delta_{L}, \alpha_{r}$ and $\alpha_{v}$ are given in the Lennard-Jones units. Parameter $\alpha_{v}$ shows the degree of the deviation from the Maxwellian distribution. The metastable state with approximately constant $T$ and $P$ exists 

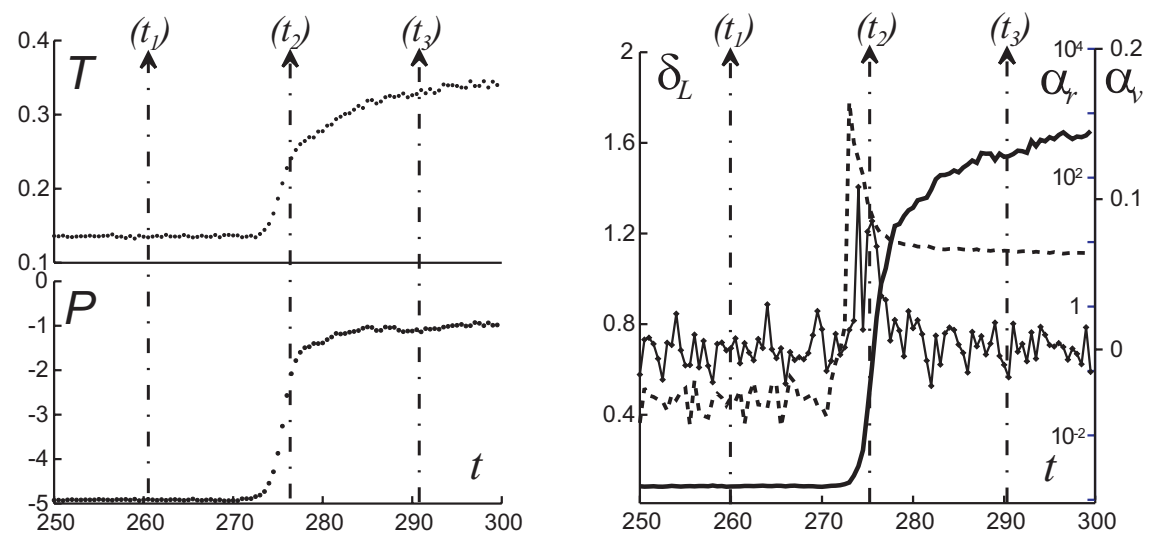

Fig. 3. The time-dependencies of $T, P$ (points), $\delta_{L}$ (bold solid), $\alpha_{r}$ (dashed) and $\alpha_{v}$ (solid) along the last interval of the MD run. $N=4000$

during $\sim 275\left(m \sigma^{2} / \epsilon\right)^{1 / 2}$ which is much greater than $t_{m}$, then spontaneous decay starts. It takes only few time units to form new more or less stationary state. The non-zero value of $\alpha_{v}$ points to the fact that the Maxwellian distribution is broken during the short decay period. The time $t_{1}$ in Fig. 3 corresponds to the long-order structure, while the structures at $t_{2}$ and $t_{3}$ reveal only short order.

The microscopic picture of the spontaneous decay is presented in Fig. 4 The decay starts with the local disordering of the crystal structure. The voids appear in the melted regions only at the next stage of the decay. Another characteristic feature of the decay is observed: the structure formed is strongly non-uniform. At least three phase states can be distinguished: crystal clusters, disordered regions and voids. In fact the final state of our MD run is not an equilibrium one, since the pressure remains to be negative till the end of our simulation (Fig. 3).
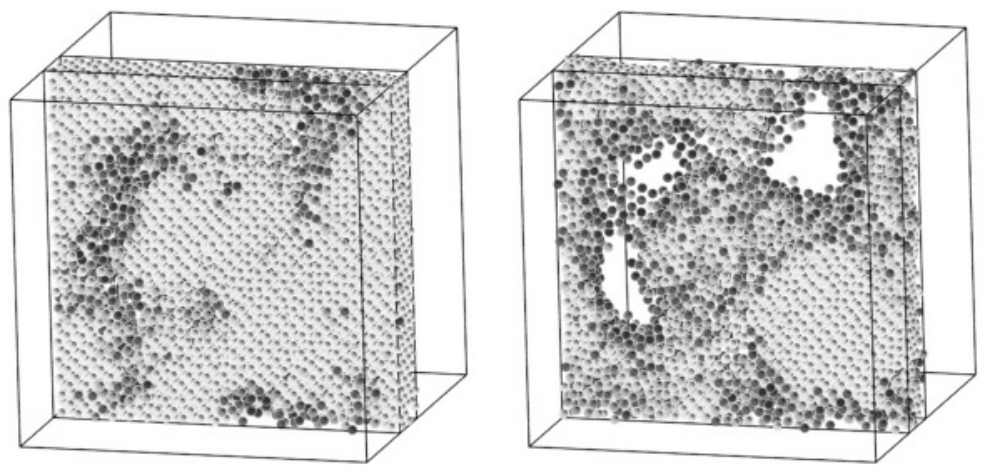

Fig. 4. The microscopic picture of the slab inside the MD cell for two moments of time corresponding to the decay process. The grey scale shows the degree of disordering. $N$ $=32000, \rho=0.8 \sigma^{-3}$ 


\section{Conclusions}

At attempt is done to formulate standard requirements to MD modeling and simulation of relaxation processes in dense media, which would be more or less universal and independent of the physical systems studied. The main features are considered which are new with respect to the simulations of equilibrium systems: 1) physically proven choice of the ensemble of initial non-equilibrium states for averaging the relaxation MD runs; 2) consideration of both first ensembledependent stage of relaxation and subsequent stage which might be ensembleindependent and remind of Boltzmann exponential relaxation; 3) calculation of dynamical memory time $t_{m}$ and comparison of $t_{m}$ with the duration of the first stage of relaxation; 4) instantaneous and time-averaged measurement procedures which are specific for studying the relaxation processes; 5) analytical extrapolation approaches to estimate relaxation times which are too long to be simulated by MD, especially for multi-scale systems. Three examples of relaxation are considered: equilibration of electrons and ions in nonideal plasmas, decay of metastable crystals under superheating or stretching.

Acknowledgments. This research is partially supported by grants NWORFBS 047.016.007, RFBS 03-11-90272v, "Integracia" U0022 and project "Parallel computations on multiprocessor computer systems" of RAS. AK, IM and VS acknowledge the support from "Dynastia" foundation and the International center of fundamental physics in Moscow. The computations were performed on the cluster granted by DAAD.

\section{References}

1. Wang, J., Li, J., Yip, S., Wolf, D., Phillpot, S. Physica A 240 (1997) 396

2. Zwicknagel, G. Contrib. Plasma Phys. 39 (1999) 155

3. Hansen, J.P., McDonald, I.R. Phys. Lett. 97A (1983) 42

4. Morozov, I.V., Norman, G.E., et al. J. Phys. A: Math. Gen. 36 (2003) 8723

5. Kuzmin, S.G., O'Neil, T.M. Phys. Plasmas 9 (2002) 3743

6. Norman, G.E., Stegailov, V.V., Valuev, A.A. Contrib. Plasma Phys. 43 (2003) 384

7. Firsov, D.A., Grigorenko, B.L., et al. Chem. Phys. Lett 338 (2001) 317

8. Stella, L., Melchionna, S. J. Chem. Phys., 109 (1998) 10115

9. Snow, C.D., Nguyen, H., Pande, V.S., Gruebele, M. Nature 420 (2002) 102

10. Tanguy, D., Mareschal, M., Lomdahl, P.S., et al. Phys. Rev. B 68 (2003) 144111

11. Morozov, I.V., Norman, G.E., Valuev, A.A. Phys. Rev. E 63 (2001) 036405

12. Norman, G.E., Stegailov, V.V. Computer Physics Comm. 147 (2002) 678

13. Gibbon, P., Pfalzner., S. Phys. Rev. E 57 (1998) 4698

14. Ebeling, W., Norman, G.E., et al. Contrib. Plasma Phys. 39 (1999) 61

15. Lifshitz, E.M., Pitaevskii, L.P. Physical kinetics (Oxford: Pergamon) (1981)

16. Riley, D., Woolsey, N.C., McSherry, D., et al. Phys. Rev. Lett. 84 (2000) 1704

17. Bonnes, D.A., Brown, J.M. Phys. Rev. Lett. 71 (1993) 2931

18. Jin, Z.H., Gumbsch, P., Lu, K., Ma, E. Phys. Rev. Lett. 87 (2001) 055703

19. Sheng-Nian Luo, Ahrens, T.J., Çă̆in T., et al. Phys. Rev. B. 68 (2003) 134206

20. Norman, G.E., Stegailov, V.V. Doklady Physics 47 (2002) 667

21. Kanel, G., Razorenov, S., Baumung, K., Singer, J. J. Appl. Phys. 90 (2001) 136 